Orthopäde $2011 \cdot 40: 793-801$

DOI 10.1007/s00132-011-1787-5

Online publiziert: 18. Juni 2011

(c) Springer-Verlag 2011

I. Falbrede ${ }^{1}$ M. Widmer ${ }^{2} \cdot$ S. Kurtz ${ }^{3}$ D. Schneidmüller ${ }^{4}$ M. Dudda ${ }^{5} \cdot$ C. Röder ${ }^{1}$

${ }^{1}$ Institut für Evaluative Forschung in der Medizin, Universität Bern

2 OBSAN Schweizerisches Gesundheitsobservatorium, Neuenburg

${ }^{3}$ Drexel University Philadelphia

${ }^{4}$ Klinikum für Unfall-, Hand- und Wiederherstellungschirurgie, Klinikum der Johann-Wolfgang-von-Goethe-Universität, Frankfurt am Main

${ }^{5}$ Klinikum Bergmannsheil, Ruhr-Universität, Bochum

\title{
Verwendungsraten von Prothesen der unteren Extremität in Deutschland und der Schweiz
}

\section{Ein Vergleich der Jahre 2005-2008}

Die totale Hüftarthroplastik ist derzeit der internationale Versorgungsstandard für degenerative Hüfterkrankungen. Obschon nationale Hüft- und/oder Knieprothesenregister in einigen europäischen Ländern implementiert wurden, haben nur wenige eine genügend hohe Dokumentationsrate, um repräsentative Aussagen über die Verwendung dieser und anderer Prothesen in den jeweiligen Ländern und Gesundheitssystemen zu machen.

\section{Hintergrund und Fragestellung}

Europäische Erhebungen haben große Unterschiede der Pro-Kopf-Ausgaben für medizinische Technologien zwischen einzelnen europäischen Ländern ergeben (http://www.eucomed.be). In den USA hat die Verwendung der totalen Hüftprothese innerhalb der letzten Dekade stark zugenommen. Es ist jedoch nicht bekannt, ob sich dieser Trend auf andere Länder übertragen lässt [12]. Ziel dieser Untersuchung war daher der detaillierte Vergleich historischer Verwendungsraten von Hüft-, Knie- und OSG-Prothesen (,total hip arthroplasty“ [THA], „total knee arthroplasty“ [TKA], OSG oberes Sprunggelenk) in der BRD und der Schweiz sowie ein Nebenvergleich mit den USA. In Ermangelung ausreichend repräsentativer
Registerdaten wurden die Datenbanken der statistischen Bundesämter (Destatis) in Deutschland und des Bundesamts für Statistik (BfS) in der Schweiz verwendet. Für die USA kamen die Daten direkt vom Autor (SK) der einschlägigen Untersuchungen. Hierbei wurde auch die von den skandinavischen Registern eingeführte Revisionslast („revision burden“) berechnet [14]. Die vorliegende Studie versuchte Antworten auf die folgenden Fragen zu finden:

- Gibt es nennenswerte Unterschiede in Verwendungsraten und Revisionslast für die Prothesen der großen Gelenke der unteren Extremität zwischen Deutschland und der Schweiz?

- Haben sich die Verwendungsraten in den vergangenen Jahren gesteigert und ergeben sich daraus stabile Trends?

- Sind die Trends und Raten in den USA ähnlich?

\section{Studiendesign und Untersuchungsmethoden}

Anfragen beim Destatis und BfS der Schweiz ergaben bereits sehr unterschiedlich strukturiertes Datenmaterial, das die wissenschaftliche Herangehensweise an die erwähnten Fragestellungen entscheidend bestimmte. Während das BfS konsistente und ausreichend vollständige Daten bis zurück ins Jahr 1998 liefern konnte, ist dies in der BRD erst ab 2005 möglich. Anfragen bei anderen potenziellen Datenlieferanten wie der Bundesqualitätssicherungsstelle BQS oder dem Bundesgesundheitsministerium ergaben keine geeigneten Möglichkeiten für die Jahre vor 2005. Diese "nachteilige" Situation wird vom Destatis dahingehend ausgeglichen, dass die Daten ab 2005 sehr detailliert vorliegen und somit Prothesensubtypen wie Femurkopf- und Oberflächenersatzprothesen („resurfacing hip“) bei der Hüfte sowie Schlittenprothesen oder interpositionelle, nicht verankerte Implantate (Metallspacer) im Bereich der Knieprothetik identifiziert und gezählt werden können, was im schweizerischen Datensatz nicht möglich ist. Daneben sind die Revisionseingriffe sehr fein differenziert und lassen kaum ein Revisionsszenario aus. Aus den vorhandenen Datensätzen wurden in Abstimmung mit einer laufenden internationalen Erhebung in Europa und den USA standardisierte Tabellen für die Jahre 2005-2008 erstellt und auch auf die OSGProthesen angewendet [11].

Die Information durch öffentliche Datenbanken ist nicht ausreichend detailliert

In Ermangelung dieser detaillierten Informationen in vielen anderen öffentlichen 


\begin{tabular}{|c|c|c|c|c|c|c|c|c|c|c|}
\hline $\begin{array}{l}\text { Vari- } \\
\text { able/ } \\
\text { Jahr }\end{array}$ & Primäre THA & $\begin{array}{l}\text { Primäre } \\
\text { Teilprothe- } \\
\text { sen }\end{array}$ & $\begin{array}{l}\text { Prothesen- } \\
\text { wechsel }\end{array}$ & $\begin{array}{l}\text { Revi- } \\
\text { sionslast } \\
(\%)\end{array}$ & $\begin{array}{l}\text { Primärrate } \\
\text { für THA } \\
\text { und Teil- } \\
\text { prothesen } \\
\left(10^{5} / \mathrm{Jahr}\right)^{\mathrm{a}}\end{array}$ & $\begin{array}{l}\text { Wechsel- } \\
\text { rate für THA } \\
\text { und Teil- } \\
\text { prothesen } \\
\left(10^{5} / \mathrm{Jahr}\right)^{\mathrm{a}}\end{array}$ & $\begin{array}{l}\text { Primäre } \\
\text { THA und } \\
\text { Teilpro- } \\
\text { thesen bei } \\
\text { Frauen (\%) }\end{array}$ & $\begin{array}{l}\text { Wechsel } \\
\text { von THA } \\
\text { und Teilpro- } \\
\text { thesen bei } \\
\text { Frauen (\%) }\end{array}$ & $\begin{array}{l}\text { Primäre THA } \\
\text { und Teil- } \\
\text { prothesen } \\
<65 \text { Jahre } \\
(\%)\end{array}$ & $\begin{array}{l}\text { Primäre THA } \\
\text { und Teilpro- } \\
\text { thesenwech- } \\
\text { sel <65 Jahre } \\
\text { (\%) }\end{array}$ \\
\hline 2005 & $159.668 / 14.781$ & $34.776 / 2990$ & $30.658 / 1966$ & $13,6 / 10,0$ & $235,8 / 238,2$ & $37,2 / 26,4$ & $64,5 / 56,1$ & $60,5 / 50,6$ & $25,8 / 31,5$ & $23,1 / 25,3$ \\
\hline 2006 & $163.664 / 15.362$ & $35.364 / 3098$ & $32.074 / 2022$ & $13,9 / 9,9$ & $241,6 / 245,7$ & $38,9 / 26,9$ & $64,1 / 55,7$ & $60,8 / 49,6$ & $25,7 / 32,1$ & $22,0 / 28,3$ \\
\hline 2007 & $168.541 / 16.656$ & $36.366 / 3091$ & $34.875 / 2049$ & $14,6 / 9,4$ & $248,0 / 260,0$ & $42,4 / 27,0$ & $63,5 / 55,5$ & $60,5 / 53,3$ & $25,3 / 32,1$ & $22,0 / 28,3$ \\
\hline 2008 & $172.460 / 16.954$ & $37.009 / 3036$ & $37.124 / 2344$ & $15,1 / 10,5$ & $254,7 / 262,7$ & $45,2 / 30,8$ & $63,2 / 56,0$ & $59,7 / 51,9$ & $25,4 / 32,7$ & $21,4 / 30,2$ \\
\hline
\end{tabular}

Tab. 2 Kennzahlen Deutschland/Schweiz für (T)KAa

\begin{tabular}{|c|c|c|c|c|c|c|c|c|c|}
\hline $\begin{array}{l}\text { Vari- } \\
\text { able/ } \\
\text { Jahr }\end{array}$ & Primäre (T)KA & $\begin{array}{l}\text { Prothesen- } \\
\text { wechsel }\end{array}$ & $\begin{array}{l}\text { Revi- } \\
\text { sionslast } \\
(\%)\end{array}$ & $\begin{array}{l}\text { Primärrate } \\
\text { für (T)KA } \\
\left(10^{5} / \mathrm{Jahr}\right)^{\mathrm{b}}\end{array}$ & $\begin{array}{l}\text { Wechselrate } \\
\text { für (T)KA } \\
\left(10^{5} / \mathrm{Jahr}\right)^{\mathrm{b}}\end{array}$ & $\begin{array}{l}\text { Primäre } \\
\text { (T)KA bei } \\
\text { Frauen (\%) }\end{array}$ & $\begin{array}{l}\text { Prothesen- } \\
\text { wechsel bei } \\
\text { Frauen (\%) }\end{array}$ & $\begin{array}{l}\text { Primäre }(T) \\
K A<65 \text { Jah- } \\
\text { re (\%) }\end{array}$ & $\begin{array}{l}\text { Prothesen- } \\
\text { wechsel } \\
<65 \text { Jahre (\%) }\end{array}$ \\
\hline 2005 & $128.920 / 10.452$ & $16.111 / 855$ & $11,1 / 7,6$ & $156,3 / 140,1$ & $19,5 / 11,5$ & $69,0 / 66,1$ & $65,3 / 62,1$ & $25,4 / 29,1$ & $28,0 / 31,9$ \\
\hline 2006 & 135.387/11.191 & $17.790 / 933$ & $11,6 / 7,7$ & $164,4 / 149,0$ & $21,6 / 12,4$ & $68,4 / 64,6$ & $65,2 / 61,3$ & $25,3 / 30,9$ & $27,8 / 31,5$ \\
\hline 2007 & $146.556 / 13.062$ & $20.260 / 1009$ & $12,1 / 7,2$ & $178,1 / 172,0$ & $24,6 / 13,3$ & $67,2 / 63,3$ & $64,4 / 62,6$ & $25,8 / 30,7$ & $27,4 / 32,3$ \\
\hline 2008 & $154.703 / 13.449$ & $22.669 / 1240$ & $12,8 / 8,4$ & $188,3 / 176,8$ & $27,6 / 16,3$ & $66,5 / 62,2$ & $63,7 / 61,9$ & $26,5 / 31,1$ & $27,7 / 36,8$ \\
\hline
\end{tabular}

aTotal- und Teilprothesen, ${ }^{b}$ pro 100.000 Personen/Jahr.

Tab. 3 Bevölkerungsstruktur Deutschland/Schweiz

\begin{tabular}{|c|c|c|c|}
\hline Variable/Jahr & $\begin{array}{l}\text { Anteil Frauen BRD/ } \\
\mathrm{CH}(\%)\end{array}$ & $\begin{array}{l}\geq 65 \text {-Jährige BRD/CH } \\
\text { (\%) }\end{array}$ & $\begin{array}{l}\text { Anteil Frauen der } \\
\geq 65 \text {-Jährigen BRD/CH (\%) }\end{array}$ \\
\hline 2005 & $51,1 / 51,0$ & $18,9 / 16,0$ & $58,9 / 58,4$ \\
\hline 2006 & $51,1 / 51,0$ & $19,5 / 16,2$ & $58,5 / 58,2$ \\
\hline 2007 & $51,0 / 50,9$ & $19,9 / 16,4$ & $58,1 / 57,9$ \\
\hline 2008 & $51,0 / 50,8$ & $20,1 / 16,6$ & $58,0 / 57,6$ \\
\hline
\end{tabular}

Datenbanken wurde nicht zwischen THA wegen degenerativer Erkrankung und wegen Fraktur des Oberschenkelhalses differenziert, womit auch die Teilprothesen miterfasst werden mussten. Des Weiteren können aus den meisten Revisionsdatensätzen nicht die Revisionen von THA und partieller HA isoliert werden, weshalb in dieser Arbeit die Revisionslast die Summe aller Wechseloperationen von Voll- und Teilprothesen geteilt durch die Summe aller primären Voll- und Teilprothesen plus die Wechseloperationen aller Voll- und Teilprothesen darstellt. Daneben wurden die Einwohnerzahl sowie Geschlechtsund Altersverteilung ermittelt. Aus diesen Kennzahlen konnte die Anzahl von Primär- Revisions- und Gesamteingriffen, Revisionslast, Primär- und Revisionsraten pro 100.00o Einwohner und Jahr sowie geschlechts- und altersspezifische Primär- und Revisionsraten berechnet werden. Für die USA wurden nur die wichtigsten Kennzahlen für die Jahre 2005 und 2008 ermittelt und aufgeführt. Die Analy- se bemüht sich um Deskription und graphische Veranschaulichung, weniger um statistische Vergleiche zwischen den Ländern. Da zudem von Vollerhebungen ausgegangen werden kann, sind für Vergleiche keine statistischen Tests erforderlich. Datenbearbeitung, Tabellenkalkulationen und Erstellung von Graphen konnten daher sämtlich mit MS Excel vorgenommen werden.

\section{Ergebnisse}

Die Kennzahlen für Deutschland und die Schweiz in den Jahren 2005-2008 sind in - Tab. 1 und 2 aufgelistet. Die Populationsstruktur beider Länder war mit Hinblick auf den Anteil Frauen in der Gesamtbevölkerung und Frauen in der Altersgruppe über 65 Jahre nahezu identisch. Es zeigte sich jedoch ein ca. 3\% höherer Anteil der über 65-Jährigen in der BRD (• Tab. 3). Die absoluten Implantationszahlen und -raten zwischen beiden Ländern zu vergleichen (• Abb. 1, 2) erscheint weniger wichtig, als Kennzahlen wie die der Revisionslast (• Abb. 3), den Quotienten zwischen Wechseloperationen und allen Operationen aufzulisten.

\section{(? Die Revisionslast für \\ Hüft- und Knieprothesen ist in Deutschland höher als in der Schweiz und USA}

Die Revisionslast für Hüftprothesen lag in der BRD bereits 2005 mit 13,6\% um 3,6\% höher, und dieser Abstand vergrößerte sich bis 2008 auf 4,6\%. Für Knieprothesen lag die Last in der BRD 2005 bei 11,1\% und damit 3,5\% höher als in der Schweiz. Bis 2008 nahm dieser Unterschied auf 4,2\% zu. In beiden Ländern lag die Revisionslast für Knieprothesen stets unter derjenigen für Hüftprothesen. In den USA lag die Hüftprothesenrevisionslast 2005 bei $11,17 \%$ und 2008 bei $11,14 \%$. Für Knieprothesen lag die Last 2005 bei $7,4 \%$ und 2008 bei $8,9 \%$.

Die Einbau- und Wechselraten pro 100.00o Personen lagen für die Hüftprothesen in der BRD unter (Primärprothesen) bzw. über (Wechseloperationen) denen der Schweiz. Mit anderen Worten werden in der BRD pro 100.00o Personen etwas weniger, nämlich über den Beobachtungszeitraum durchschnittlich 245 Primärhüften gegenüber 252 in $\mathrm{CH}$ implantiert, aber 42 Wechselope- 


\section{Falbrede · M. Widmer · S. Kurtz · D. Schneidmüller · M. Dudda · C. Röder Verwendungsraten von Prothesen der unteren Extremität in Deutschland und der Schweiz. Ein Vergleich der Jahre 2005-2008}

\section{Zusammenfassung}

Hintergrund. In den USA hat die Verwendung der totalen Hüftprothese innerhalb der letzten Dekade stark zugenommen. Es ist jedoch nicht bekannt, ob sich dieser Trend auf andere Länder übertragen lässt. Ziel dieser Untersuchung war daher der detaillierte Vergleich der Verwendungsraten von Hüft-, Knie- und OSG-Prothesen in der BRD und der Schweiz im Zeitraum 2005-2008 und ein Sekundärvergleich mit den USA.

Patienten und Methoden. Aus Daten der beiden statistischen Bundesämter wurden die Einwohnerzahl, Geschlechts- und Altersverteilung sowie die Anzahl von Primärund Revisionseingriffen ermittelt. Aus diesen Kennzahlen konnte die Anzahl von Primär-, Revisions- und Gesamteingriffen, der Revisionslast, von Primär- und Revisionsraten pro 100.000 Einwohner und Jahr sowie geschlechts- und altersspezifische Primär- und Revisionsraten berechnet werden. Ein im Ver- gleich dazu reduzierter Datensatz aus den USA stammt direkt vom Autor der entsprechenden Auswertungen.

Ergebnisse. In Deutschland, der Schweiz und den USA stieg die Anzahl der Vollund Teilhüftprothesenimplantationen pro 100.000 Personen von $235,8,238,2$ und 116,8 im Jahr 2005 auf 254,7, 262,7 und 127,3 an. Für Voll- und Teilknieprothesen lagen die Raten bei $156,3,140,1$ und 178,2 Implantationen in 2005 und 188,3, 176,8 und 213,6 in 2008. Die Revisionslast für Hüftprothesen lag in der BRD in 2005 mit 13,6\% um 3,6\% höher als in der Schweiz und betrug $11,2 \%$ in den USA. In 2008 lag sie mit 15,1\% um 4,6\% höher als in der Schweiz und betrug wiederum $11,2 \%$ in den USA. Für Knieprothesen lag die Last in der BRD in 2005 bei $11,1 \%$ und damit $3,5 \%$ höher als in der Schweiz; in den USA betrug sie $7,4 \%$. In 2008 lag sie bei $12,8 \%$ und somit 4,2\% höher als in der Schweiz und be- trug 8,9\% in den USA. In allen 3 Ländern lag die Revisionslast für Knieprothesen stets unter derjenigen für Hüftprothesen.

Schlussfolgerung. In allen 3 Ländern stiegen die Primärraten für Hüft- und Knieprothesen an, diejenigen für Knieprothesen stärker. Die Revisionslasten lagen in 2008 für beide Prothesentypen in der BRD am höchsten. In der Schweiz gab es eine vorübergehende Senkung der Revisionslasten und erst ab 2007 wieder einen Anstieg. Die Verwendungsrate von Hüftprothesen pro 100.000 Einwohner lag in den USA stark unter den Raten der BRD und der Schweiz, für Knieprothesen lag sie leicht darüber.

\section{Schlüsselwörter}

Verwendungsraten · Revisionslast . Hüftgelenkprothese · Kniegelenkprothese · Prothese des oberen Sprunggelenks

\section{Utilization rates of lower extremity prostheses in Germany and Switzerland. A comparison of the years 2005-2008}

\section{Abstract}

Background. In the United States the use of total hip arthroplasty (THA) has substantially increased over the last decade. It is not known, however, if this trend can be applied to other countries as well. The aim of the current study was therefore a detailed comparison of hip, knee, and ankle arthroplasty utilization rates in Germany and Switzerland in the years 2005-2008 and a secondary comparison with the United States.

Patients and methods. Based on datasets from the national statistical offices the number of inhabitants, gender and age distributions and the number of primary and revision surgeries were determined. These figures served for calculating primary, revision and overall surgical volumes, revision burden, primary and revision rates per 100,000 inhabitants, gender and age-specific primary and revision rates. A comparably smaller dataset was provided for the respective US analyses.
Results. In Germany, Switzerland and the US the number of implanted total and partial hip arthroplasties per 100,000 inhabitants rose from 235.8, 238.2 and 116.8 in 2005 to $254.7,262.7$ and 127.3 in 2008 , respectively. For total and partial knee arthroplasty the rates were $156.3,140.1$ and 178.2 implantations in 2005 and $188.3,176.8$ and 213.6 in 2008 , respectively. With $13.6 \%$ the revision burden in Germany was 3.6\% higher than in Switzerland and accounted for $11.2 \%$ in the US. In 2008 it was $15.1 \%$ in Germany, was hence $4.6 \%$ higher than in Switzerland and remained stable at $11.2 \%$ in the US. For knee replacements the 2005 German revision burden was $11.1 \%$ which was $3.5 \%$ higher than in Switzerland and was $7.4 \%$ in the US. In 2008 it was $12.8 \%$ in Germany and $4.2 \%$ lower in Switzerland and in the US it accounted for $8.9 \%$. In all three countries the revision burden for knee arthroplasty was constantly lower than for hip arthroplasty.

Conclusion. In all three countries the primary rates for hip and knee replacements rose over the years but those for knee arthroplasty to a higher extent. The 2008 revision burden was highest in Germany for both types of arthroplasty. In Switzerland there was a transient revision burden decrease with a new increase from the year 2007 onwards. The US hip replacement utilization rates per 100,000 inhabitants were considerably lower than those in Germany and Switzerland and for knee replacements they were slightly higher.

Keywords

Utilization rate $\cdot$ Revision burden $\cdot$ Hip prosthesis $\cdot$ Knee prosthesis $\cdot$ Ankle prosthesis 


\section{Originalien}

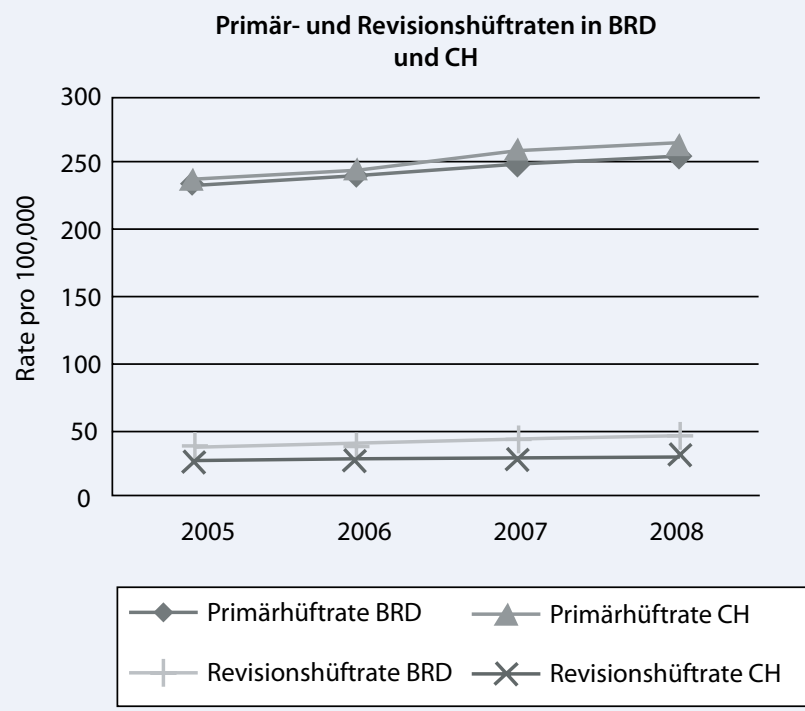

Abb. $1 \Delta$ Primärimplantations- und Revisionsraten von Hüftprothesen in Deutschland und der Schweiz

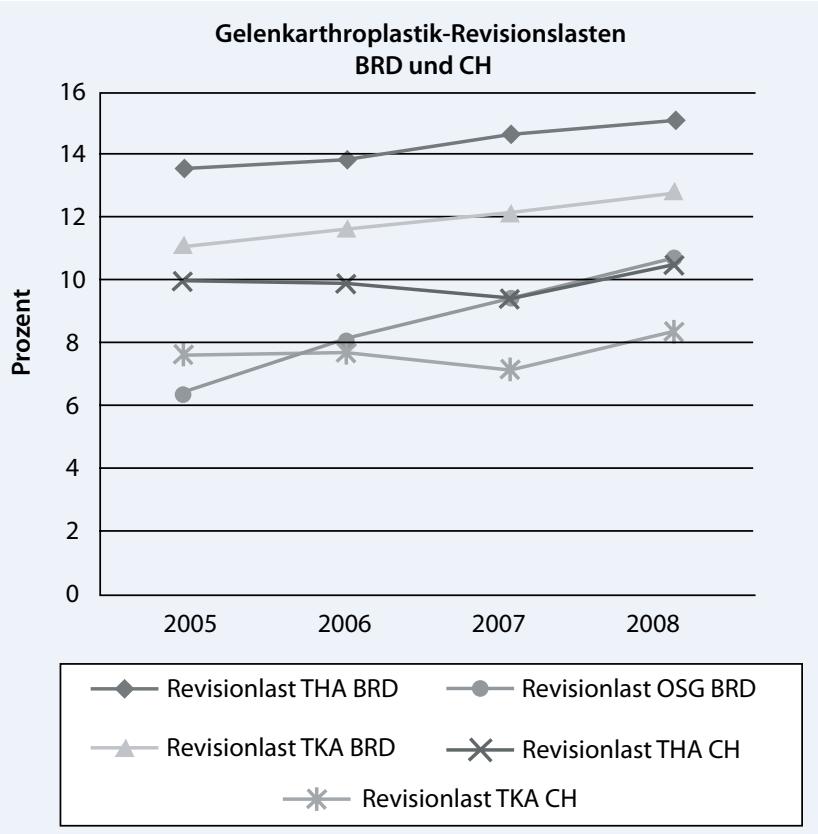

Abb. $3 \Delta$ Revisionslasten für Hüft- und Knieprothesen in Deutschland und der Schweiz. Bemerkenswert ist der nicht konstante Trend für beide Prothesentypen in CH. Von 2005-2007 sanken die Lasten und stiegen erst in 2008 wieder auf ein höheres Niveau als im Jahr 2005 an. Die Graphik zeigt zudem den vergleichsweise stärksten Anstieg der Revisionslast für die OSG-Prothesen in der BRD. OSG oberes Sprunggelenk

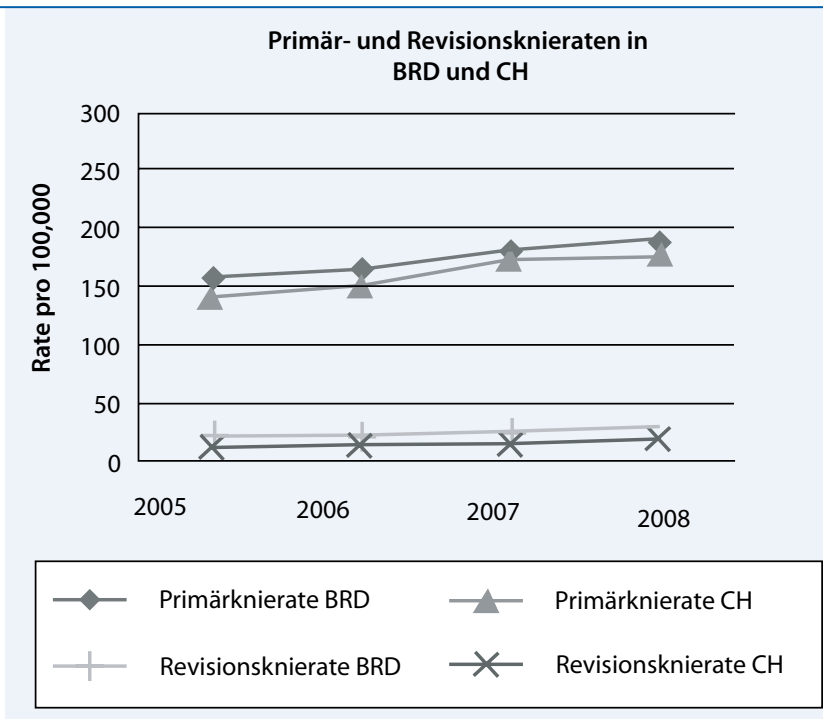

Abb. $2 \Delta$ Primärimplantations- und Revisionsraten von Knieprothesen in Deutschland und der Schweiz

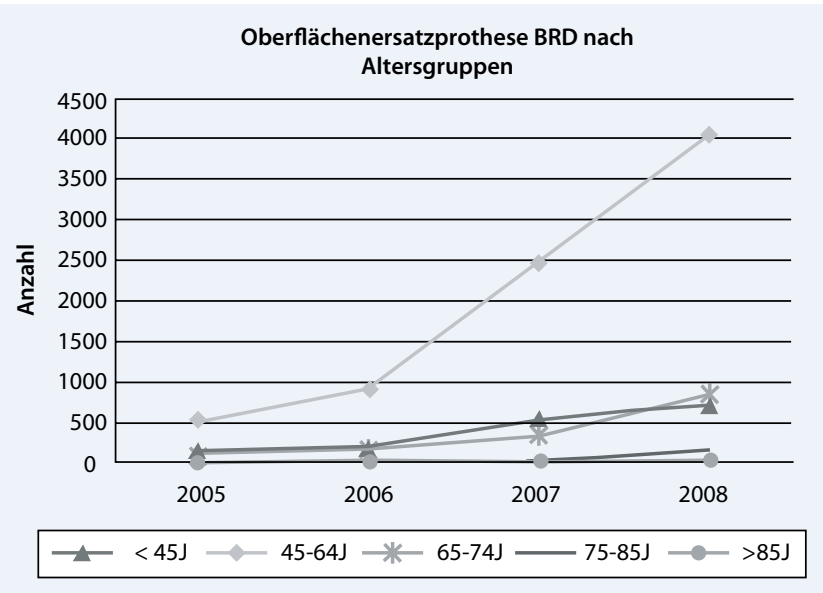

Abb. $4 \Delta$ Verbreitung der Oberflächenersatzprothese in der BRD rationen gegenüber 28 in $\mathrm{CH}$ durchgeführt. Dementsprechend war die oben genannte Revisionslast in der BRD für die Hüften höher. In der Knieprothetik wurden über den Beobachtungszeitraum durchschnittlich 172 Primärprothesen in der BRD gegenüber 159 in $\mathrm{CH}$ pro 100.000 Personen implantiert sowie 23 Wechseloperationen gegenüber 13 in der Schweiz durchgeführt, was die Revisionslast wiederum in der BRD höher ausfallen ließ.

In den USA wurden in $2005(116,9)$ und $2008(127,3)$ nur ca. die Hälfte an Hüftprothesen pro 100.000 Einwohner implantiert wie in der BRD und der Schweiz, dafür aber 15-25\% mehr Knieprothesen $(178,2$ und 213,6$)$.
Die Verwendungsraten und Gesamtzahlen für OSG-Prothesen waren im Vergleich zu denen für THA und TKA gering. Von 2005-2008 wurden in der BRD 10671145-1268-1356 primäre OSG-Prothesen eingebaut und 73-101-131-163 Wechseloperationen durchgeführt. Dies entspricht Revisionslasten von 6,4-8,1-9,4$10,7 \%$, primären OSG-Implantationsra- 
ten von 1,3-1,4-1,5-1,7 und Wechselraten von 0,1-0,1-0,2-0,2/100.00o Personen. In der Schweiz wurden im Beobachtungszeitraum 238-226-303-281 OSG-Prothesen eingebaut, was Implantationsraten von 3,2-3-4-3,7/100.0oo entspricht. Wechselprozeduren für OSG-Prothesen werden in der Schweiz noch nicht kodiert. Für die USA liegen keine Vergleichszahlen vor.

\section{(? In Deutschland erhalten mehr Frauen und eher ältere Patienten eine Prothese}

Bezüglich der Alters- und Geschlechtsverteilung gab es deutliche Unterschiede zwischen beiden Ländern. Während der Prozentsatz weiblicher Patienten mit einer primären Hüftprothese in der BRD ca. 8\% höher lag und der mit einer Wechseloperation um 9\%, lag der Anteil der Patienten unter 65 Jahren für die Primär- und Wechseloperationen in der Schweiz um ca. $6-7 \%$ höher. Diese Verteilungen trafen in abgeschwächter Form auch für die Knieprothesen zu. Etwa 3-4\% mehr Frauen erhielten Primärknie- oder Wechseloperationen in der BRD; ca. 4-6\% mehr Patienten unter 65 Jahren erhielten Primärknie- oder Wechseloperationen in der Schweiz.

Am Beispiel der Oberflächenersatzprothese wird ersichtlich, wie sich neue Therapien im Markt durchsetzen (- Abb. 4). In der Altersgruppe 4564 Jahre hat sich der Einbau dieses Prothesentyps verachtfacht, ohne Unterschied zwischen den Geschlechtern (nicht aus der Abbildung ersichtlich). Derartige Trends für Hüftprothesensubtypen sind aus dem schweizerischen Datensatz nicht ableitbar. Die Marktpenetration kann aber auch alters- und geschlechtsspezifisch unterschiedlich sein (- Abb. 5a,b). Die Schlittenprothesenverwendung nahm beispielsweise bei den Frauen nur in der Altersgruppe 4564 Jahre zu, sonst sind eher abnehmende Implantationszahlen oder quasi Stagnationen ersichtlich. Bei den Männern hingegen nahm der Einbau sowohl in den Altersgruppen 45-64 als auch 65-74 Jahre zu. - Abb. 6 zeigt starke Zunahmen für den Patellaersatz in allen Altersgruppen unter 85 Jahren. Die Zunahmen sind stärker als die für die Knietotalprothesen.

\title{
Hier steht eine Anzeige.
}

\author{
Springer
}




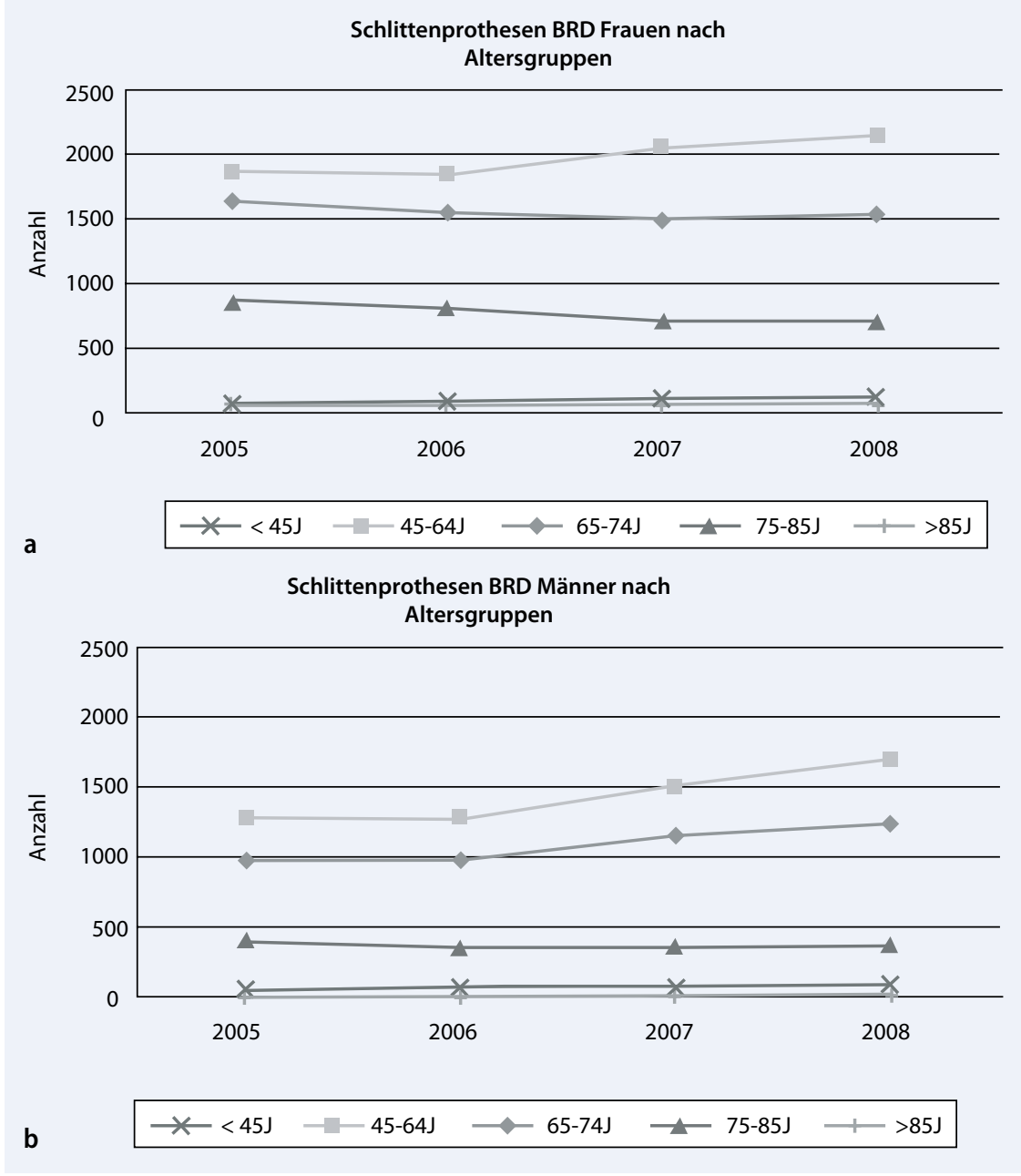

tationsraten für die Hüftprothesen in der Schweiz, für die Knieprothesen aber in der BRD. Zieht man die beiden punktuellen Messungen aus den USA in Betracht, findet man deutlich niedrigere Primärimplantationsraten für die Hüftprothesen, aber leicht höhere bei den Knieprothesen. Die Revisionsraten waren für beide Prothesentypen in der BRD höher. Die Zunahme der Primärraten verlief in beiden Ländern nahezu parallel, die der Revisionsraten ebenfalls, jedoch weniger stark.

\section{( In der Schweiz ist eine temporäre Abnahme der Revisionslasten feststellbar}

Auch die Revisionslasten lagen in der BRD für beide Prothesentypen höher und folgten stetig zunehmenden Trends, während diese in der Schweiz zumindest in den Jahren 2005-2007 abnahmen. Die Revisionslasten der USA ähnelten für die Knieprothesen denen der Schweiz, für die Hüftprothesen lagen sie zwischen der Schweiz und der BRD und blieben von 2005-2008 nahezu stabil. Die OSG-Primärimplantationsraten lagen in der BRD zwischen $1,3-1,7 \%$ und in der Schweiz ca. doppelt so hoch. Die Revisionslasten für die OSG-Prothesen verzeichneten in der BRD die stärkste Zunahme aller untersuchten Prothesen. Bei den Hüftund Knieprothesen konnte man generell eine leicht höhere Rate weiblicher Patienten in der BRD beobachten, während in der Schweiz die Rate von Patienten unter 65 Jahren höher war.

Bezüglich der eingangs erwähnten Fragestellungen nach nennenswerten Unterschieden hinsichtlich Verwendungsraten und Revisionslast für die Prothesen der großen Gelenke der unteren Extremität zwischen Deutschland und der Schweiz sind v. a. die Revisionslasten hervorzuheben. Diese liegen sowohl für Hüft- als auch Knieprothesen deutlich höher in der BRD und weisen zusätzlich eine stetige Zunahme über die Zeit auf. Dies bedeutet, dass der Anteil an Wechseleingriffen gemessen an der Gesamtzahl aller durchgeführten Primär- und Wechseloperationen in der BRD höher ist und weiter zunimmt. In der Schweiz dagegen hat zumindest von 2005-2007 die Revisionslast 
sogar abgenommen, um dann 2008 wieder zuzunehmen.

Im Gegensatz dazu liegen die pro 100.00o Einwohner durchgeführten Primär- und Revisionsraten sowohl für Hüftals auch Knieprothesen in beiden Ländern bzgl. ihrer Höhe enger beieinander und folgen auch ähnlichen Trends, nämlich leichten und konstanten Zunahmen bei den Primärimplantationsraten und nur geringen Zunahmen bei den Revisionsraten. Bezieht man die US-Zahlen mit ein, fallen v. a. die deutlich niedrigeren Primärimplantationsraten für die Hüftprothesen und die leicht höheren Raten für die Knieprothesen auf. Bezüglich der Revisionslasten ergibt sich ein gemischtes Bild mit stabilen Hüftrevisionslasten über dem Niveau der Schweiz und unter der BRD und ähnlich niedrigen Lasten wie denen der Schweiz für die Knieprothesen.

Es gibt zahlreiche internationale Studien, die konsistent sind mit unseren Ergebnissen und eine Zunahme der primären Implantations- und Wechselraten für Prothesen der unteren Extremität belegen $[4,9,10]$. Für die USA beschreiben Kurtz et al. [9] über einen Zeitraum von 1990-2002 einen Zuwachs von $46 \% / 100.000$ Personen für primäre THA, wobei sich die entsprechende Rate für primäre Knieprothesen in diesen Jahren fast verdreifachte. Gleichzeitig stiegen die Revisionsraten für THA und TKA um 3,7 bzw. 5,4 Eingriffe/10o.ooo Personen/Dekade (entsprechend 6o bzw. 166\%) an.

Ähnlich unseren Ergebnissen waren die Raten aller 4 operativen Eingriffe (primäre THA und TKA sowie Revisions-THA und -TKA) für Frauen in den USA signifikant höher als für Männer. Auch altersadjustierte Primär- und Revisionsraten zeigten in dieser Studie in allen Altersgruppen einen von Jahr zu Jahr zunehmenden stabilen Trend. Die Revisionslast für Hüftprothesen in den USA beschreiben Kurtz et al. [9] über die Jahre als relativ konstant. Sie lag mit im Mittel $17,5 \%$ mehr als doppelt so hoch wie die für Knieprothesen, wobei bei den damaligen Berechnungen die primären Teilprothesen nicht berücksichtigt wurden, was den Nenner bei der Revisionslastberechnung verkleinert und die Last damit rechnerisch erhöht. Damit und unter Berücksichtigung möglicher Unschärfen bei den
Berechnungen aus den offensichtlich gewordenen Gründen der Teilprotheseninklusion oder -exklusion war die Hüftrevisionslast in den USA zwischen 1990 und 2002 ähnlich hoch wie die Hüftrevisionslasten in einem Teil der Länder mit etablierten Hüft- und Knieregistern wie Norwegen, Finnland und Australien (15-18,3\%). In Kanada und Schweden lagen die Hüftrevisionslasten mit 13,1 bzw. $11 \%$ deutlich niedriger. In ähnlicher Weise verhielt es sich mit den Knierevisionslasten, bei denen sich die USA im Beobachtungszeitraum im Bereich der dokumentierten Knierevisionslasten von Norwegen, Schweden und Finnland bewegte $(7,2-8,0 \%)$. Die kanadische Knierevisionslast lag mit $6,1 \%$ etwas niedriger, die australische mit 10,8\% etwas höher.

\section{จ Immer jüngere Patienten erhalten Gelenkprothesen}

Neben dem allgemeinen Anstieg der TKA-Verwendungsraten zeigen z. B. Khatod et al. [8] einen insbesondere hohen prozentualen Anstieg der operativen Knieeingriffe in jüngeren Altersgruppen. So stieg die Rate der TKA in der Altersgruppe unter 65 Jahren um 8,8\% jährlich, wogegen die Rate in der Altersgruppe über 65 Jahre um 2,1\%/Jahr anstieg. Kurtz et al. [9] verdeutlichen eine ähnliche Entwicklung auch für die Hüftprothetik. In der Patientenpopulation im Alter von 4584 Jahren, die mit primärer THA behandelt wurden, stieg der relative Anteil derjenigen, die zwischen 45 und 64 Jahre alt waren, von 28 auf $40 \%$ zwischen 1990 und 2002. Im Patientenkollektiv mit primärer TKA stieg der relative Anteil derjenigen in der Altersgruppe 45-64 Jahre von 26 auf $36 \%$ über den selben Zeitraum. Dagegen kommen Culliford et al. [4] in ihren Untersuchungen der britischen Daten von 1991-2006 zu dem Schluss, dass das verstärkte Implantieren von Knieprothesen bei jüngeren Patienten eine Fehlperzeption sei, da sich das Durchschnittsalter der TKA-Empfänger über den Beobachtungszeitraum nicht geändert hatte. Diese Befunde können aber auch durchaus von der Art des britischen Gesundheitssystems mit mehr Planung und Rationierung beeinflusst sein.
Nach den Prognosen von Kurtz et al. [10] bzgl. der primären und Revisionseingriffe in der Hüft- und Kniegelenkarthroplastik werden diese Trends anhalten. So wird der Bedarf an primären THA in den Vereinigten Staaten schätzungsweise von 2005-2030 um 174\% auf 572.00o Eingriffe anwachsen und der für primäre TKA um 673\% auf 3,48 Mio. Eingriffe. Für den gleichen Zeitraum prognostizieren Kurtz et al. einen Zuwachs der Hüft- und Knierevisionseingriffe von 137 bzw. 601\%.

Eine alternde Bevölkerung, höhere körperliche Aktivität, eine um 25\% erhöhte Lebenserwartung und nicht zuletzt eine stark zunehmende Prävalenz von Adipositas in der westlichen Welt, die schon bei immer jüngeren Patienten zu degenerativen Gelenkerkrankungen führt, sind zusammen mit einer zunehmenden Effizienz der operativen Eingriffe bzgl. Liegezeiten, Komplikationen, Rehabilitation und Prothesenstandzeiten Hauptfaktoren der ansteigenden Implantationsraten der Hüft- und Kniegelenkprothesen $[3,5,16]$. Die demographischen Veränderungen mit einem höheren Kollektiv an osteoarthrosegefährdeten jüngeren Patienten, die ihr künstliches Gelenk überleben, werden trotz der Verbesserungen im Prothesenmaterial und -design und Fortschritten bei den operativen Techniken auch zu ansteigenden Revisionseingriffen führen $[8,10]$. Dies würde wiederum erklären, warum sich keine Reduktion der Revisionslast verzeichnen lässt, sondern eher sogar Steigerungen. Diese Kenngröße wird als Vergleichsparameter der relativen Häufigkeit von Wechseleingriffen in verschiedenen Ländern eingesetzt. Eine internationale vergleichende Untersuchung mit Datensätzen aus dem Zeitraum 2006-2008 aus 13 europäischen Ländern sowie Kanada und den USA zeigt eine Spanne von Hüftrevisionslasten von $7,3 \%$ (Rumänien) bis $16,6 \%$ (Spanien) mit einem internationalen Schnitt von $12,9 \%$. Hier liegen Deutschland mit $14,6 \%$ in 2007 knapp über dem Durchschnitt und die Schweiz mit 9,4\% deutlich darunter [11]. Unschärfen bestimmt durch Kodierungen, Definitionen (THA, Teilprothese, degenerative Osteoarthrose, traumatische Oberschenkelhals- 
fraktur) oder unvollständige Statistiken müssen bei diesen Untersuchungen jedoch immer berücksichtigt werden. Bei den Knierevisionslasten mit einer Spanne von $2,7 \%$ (Rumänien) bis $12,1 \%$ (BRD) und einem internationalen Durchschnitt von $8,3 \%$ liegt die Schweiz genau im Schnitt, während die BRD das Schlusslicht darstellt [11].

Ob sich hier die präziseste Datenerfassung gegen den eigenen Berufsstand wendet oder tatsächlich hohe Revisionsraten vorliegen, ist mit den uns verfügbaren Daten nicht mit letzter Sicherheit zu klären.

\section{() Weibliches Geschlecht und höheres Alter schützen vor Hüftprothesenlockerung}

Studien von Röder et al. [17] und Münger et al. [16] et al. dokumentieren, dass bestimmte Patientencharakteristika wie Alter, Geschlecht, Adipositas und Mobilität Risikofaktoren darstellen, die Einfluss haben auf eine vorzeitige aseptische Pfannen- und Schaftlockerung nach THA und somit einen Wechseleingriff bedingen. In beiden Studien zeigte das weibliche Geschlecht ein signifikant niedrigeres Risiko einer Schaftlockerung und auch einer Pfannenlockerung sowohl bei der zementierten als auch unzementierten Komponentenfixation. Ebenso sank das Risiko einer Prothesenlockerung mit jedem zusätzlichen Lebensjahr des Patienten zum Zeitpunkt der Operation. Das bedeutet, männliches Geschlecht und jüngeres Alter stellen Risikofaktoren bzgl. Pfannenund Schaftlockerung dar. Verschiedene andere Studien lieferten ähnliche Ergebnisse $[2,13,21]$. Studien über den Einfluss von Patientencharakteristika auf eine TKA-Lockerung liefern dagegen weniger eindeutige und auch widersprüchliche Ergebnisse [1].

Betrachtet man die patientenbezogenen demographischen Faktoren wie jüngeres Alter und männliches Geschlecht, die das Risiko eines Hüftrevisionseingriffs erhöhen, stellt sich heraus, dass Deutschland im Vergleich zur Schweiz sogar über ein Patientenkollektiv mit weniger Risikokandidaten verfügt. Wie bereits oben dargelegt, findet man in Deutschland einen höheren Prozentsatz weiblicher $\mathrm{Pa}$ - tienten bei den primären und RevisionsTHA und -TKA. Für alle 4 Eingriffe lag der Anteil der Patienten unter 65 Jahren in der Schweiz höher. Das heißt, würden wir unsere Daten nach Alter und Geschlecht korrigieren, würden die deutschen Revisionslasten vergleichsweise noch ungünstiger abschneiden. Erklärungen hierfür bleiben z. Z. noch Mutmaßungen und bedürfen weiterer fundierter wissenschaftlicher Untersuchungen. Ein Erklärungsansatz könnten unterschiedliche Operationsvolumina für die Majorität der Operateure in der $\mathrm{BRD}$ und $\mathrm{CH}$ sein, für die jedoch keine repräsentativen Zahlen vorliegen.

Mehrere amerikanische Studien haben diese Zusammenhänge untersucht. Manley et al. [15] dokumentieren, dass die Wahrscheinlichkeit eines Revisionseingriffs bis zu 6 Monate nach dem primären Einsatz einer Hüft- oder Kniegelenkprothese vom Eingriffsvolumen des Operateurs abhängig ist. So hatten $\mathrm{Pa}$ tienten, die von Orthopäden mit geringeren Eingriffsvolumina/Jahr (bis 25) operiert wurden, ein signifikant höheres Risiko für einen Wechseleingriff als Patienten von Orthopäden mit höheren Eingriffsvolumina/Jahr. Das pro Klinik gesamte operierte Volumen hatte dabei keinen Einfluss auf die Revisionsrate, wohl aber auf die Mortalitäts-, Hüftgelenksluxations- und perioperative Komplikationsrate $[6,20]$. In der BRD gibt es im Gegensatz zur Schweiz eine Mindestmengenregelung für Knieprothesen seit dem Jahr 2005, nach der ein Operateur mindestens 50 Implantationen/ Jahr vornehmen muss. Für die Hüftprothesen gibt es noch keine solche Bestimmung. Schräder et al. [18] konnten jedoch aus deutschen Routinedaten keinen eindeutigen Schwellenwert ableiten, der mit ausreichender Evidenzgrundlage einen guten von einem schlechten Operateur hätte unterscheiden können. Obschon es in der Literatur eine positive Korrelation zwischen Operationsvolumen und bestimmten Ergebnisqualitätsparametern gibt, kann auch hier kein einheitlicher Zusammenhang zwischen hoher Fallmenge und guter Ergebnisqualität aus den deutschen Routinedaten abgeleitet werden [19].
Die steigenden Implantationsraten der Prothesen der unteren Extremität und die damit einhergehenden höheren Wechselraten haben erhebliche Auswirkungen auf unser Gesundheitssystem. Revisionseingriffe implizieren zusätzliche Kosten, höhere Komplikationsraten, längere Operationszeiten und suboptimalere Ergebnisse. Es wird eine Herausforderung sein, genügend qualifizierte Orthopäden und ausreichende Mittel und Kapazitäten bereitzustellen, um diesem erhöhten quantitativen Bedarf gerecht zu werden $[7,10]$. Alle diese Ausführungen und der in dieser Arbeit offenkundig gewordene Mangel an detaillierten klinischen und auch Outcomedaten unterstreichen den Bedarf an der Etablierung eines nationalen Gelenkregisters, wie es bereits in zahlreichen anderen Ländern existiert (Skandinavien, Australien, Kanada). Die Transparenz von Informationen und damit potenziell die Qualität und Effizienz von Hüftund Knieprothesen wird durch die zentrale Dokumentation folgender prospektiver Daten erhöht:

- Diagnose,

- patientendemographische Faktoren,

- Art des Eingriffs,

- Prothesentyp (mit Artikel- und

Seriennummer),

- operative Techniken und Komplikationen,

- klinische Ergebnisse (Outcome) bei Entlassung und im Verlauf,

- Kosten,

- Revisionen in ähnlichem Detail.

Mit den aus der Routinearbeit abgeleiteten Daten eines Gelenkregisters könnten suboptimale Implantate und Operationsverfahren sowie Patienten mit hohen Risikofaktoren für Revisionseingriffe und Komplikationen identifiziert, regionale und internationale Unterschiede aufgedeckt und neue Technologien beurteilt werden. Sowohl die Gesundheitspolitik als auch Orthopäden, Krankenhäuser, Kostenträger und nicht zuletzt die Patienten könnten sich an evidenzbasierter Information orientieren und einen optimalen individuellen Behandlungsplan aufstellen. Durch Überweisung bestimmter Patienten mit einem erhöhten Versagensrisiko an Schwerpunktzentren könn- 
ten Revisionsraten potenziell zusätzlich gesenkt werden.

\section{Korrespondenzadresse \\ PD Dr. C. Röder}

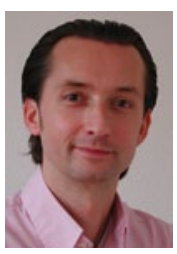

Institut für Evaluative

Forschung in der Medizin,

Universität Bern

Stauffacherstr.78, CH-3014 Bern

Schweiz

christoph.roeder@memcenter.

unibe.ch

Interessenkonflikt. Der korrespondierende Autor gibt an, dass kein Interessenkonflikt besteht.

\section{Literatur}

1. Sculco T, Martucci E (eds) (2002) Total knee arthroplasty, chapter 16. Springer, Berlin Heidelberg New York

2. Berry DJ, Harmsen WS, Cabanela ME et al (2002) Twenty-five-year survivorship of two thousand consecutive primary Charnley total hip replacements: factors affecting survivorship of acetabular and femoral components. J Bone Joint Surg [Am] 84-A:171-177

3. Crowninshield RD, Rosenberg AG, Sporer SM (2006) Changing demographics of patients with total joint replacement. Clin Orthop Relat Res 443:266-272

4. Culliford DJ, Maskell J, Beard DJ et al (2010) Temporal trends in hip and knee replacement in the United Kingdom: 1991 to 2006. J Bone Joint Surg [Br] 92:130-135

5. Foran JR, Mont MA, Rajadhyaksha AD et al (2004) Total knee arthroplasty in obese patients: a comparison with a matched control group. J Arthroplasty 19:817-824

6. Katz JN, Mahomed NN, Baron JA et al (2007) Association of hospital and surgeon procedure volume with patient-centered outcomes of total knee replacement in a population-based cohort of patients age 65 years and older. Arthritis Rheum 56:568-574

7. Kelly MP, Bozic KJ (2009) Cost drivers in total hip arthroplasty: effects of procedure volume and implant selling price. Am J Orthop 38:E1-E4

8. Khatod M, Inacio M, Paxton EW et al (2008) Knee replacement: epidemiology, outcomes, and trends in Southern California: 17,080 replacements from 1995 through 2004. Acta Orthop 79:812-819

9. Kurtz S, Mowat F, Ong K et al (2005) Prevalence of primary and revision total hip and knee arthroplasty in the United States from 1990 through 2002. J Bone Joint Surg [Am] 87:1487-1497

10. Kurtz S, Ong K, Lau E et al (2007) Projections of primary and revision hip and knee arthroplasty in the United States from 2005 to 2030. J Bone Joint Surg [Am] 89:780-785

11. Kurtz S, Röder C, Lau E et al (2010) International survey of primary and revision total hip replacement. 56th annual meeting of the orthopaedic research society. New Orleans, USA

12. Kurtz SM, Ong KL, Schmier J et al (2007) Future clinical and economic impact of revision total hip and knee arthroplasty. J Bone Joint Surg [Am] 89(Suppl 3):144-151
13. Mahomed NN, Barrett JA, Katz JN et al (2003) Rates and outcomes of primary and revision total hip replacement in the United States medicare population. J Bone Joint Surg [Am] 85-A:27-32

14. Malchau $H$, Herberts $P$, Eisler T et al (2002) The Swedish total hip replacement register. J Bone Joint Surg [Am] 84-A(Suppl 2):2-20

15. Manley M, Ong K, Lau E et al (2008) Effect of volume on total hip arthroplasty revision rates in the United States medicare population. J Bone Joint Surg [Am] 90:2446-2451

16. Münger $P$, Röder $C$, Ackermann-Liebrich $U$ et al (2006) Patient-related risk factors leading to aseptic stem loosening in total hip arthroplasty: a casecontrol study of 5,035 patients. Acta Orthop 77:567-574

17. Röder C, Bach B, Berry DJ et al (2010) Obesity, age, sex, diagnosis, and fixation mode differently affect early cup failure in total hip arthroplasty: a matched case-control study of 4420 patients. J Bone Joint Surg Am 92:1954-1963

18. Schräder P, Grouven U, Bender R (2007) Is it possible to calculate minimum provider volumes for total knee replacement using routine data? Results of a threshold value analysis of German quality assurance data for inpatient treatment. Orthopade 36:570-576

19. Schräder P, Rath T (2007) Volume-outcome-relationship in total hip replacement-literature review and model calculation of the health care situation Z Orthop Unfall 145:281-290

20. Shervin N, Rubash HE, Katz JN (2007) Orthopaedic procedure volume and patient outcomes: a systematic literature review. Clin Orthop Relat Res 457:35-41

21. Young NL, Cheah D, Waddell JP et al (1998) Patient characteristics that affect the outcome of total hip arthroplasty: a review. Can J Surg 41:188-195

\section{Bildgebung vor und nach arthroskopischen Eingriffen}

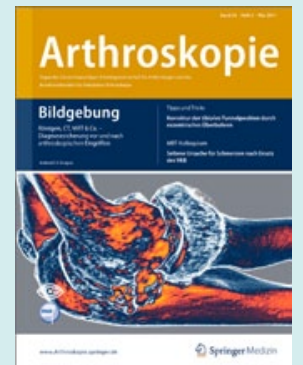

Die Arthroskopie stellt nach wie vor den Goldstandard zur Beurteilung der rein intraartikulären Pathologien der verschiedenen Gelenke dar. Dennoch ist die

Arthroskopie als operativer invasiver Eingriff nicht das primäre Verfahren zur Evaluierung von Veränderungen in einem Gelenk. Aber bei welcher klinischen Verdachtsdiagnose ist welches nichtinvasive Verfahren zur präoperativen Diagnosesicherung am besten geeignet? Und wie formuliert man die Fragestellung für die Radiologie, so dass die betroffene Pathologie optimal bildgebend dargestellt werden kann?

Über diese Punkte und weitere Fragestellungen im Zusammenhang mit der „Bildgebung vor und nach arthroskopischen Eingriffen“ informiert die Zeitschrift Arthroskopie in Heft 2/2011.

Die Schwerpunkte des Leitthemenheftes

sind:

- Schnittbilddiagnostik vor und nach Arthroskopie

- Bildgebung vor und nach arthroskopischen Eingriffen an der Schulter

- Schnittbilddiagnostik des Ellenbogens und der Hand

- Korrelation der Bildgebung mit der Arthroskopie des Hüftgelenks

- Bildgebung postarthroskopischer Komplikationen nach Knieverletzungen

Bestellen Sie diese Ausgabe zum Preis von $61,-€$ bei Springer Customer Service Center Kundenservice Zeitschriften

Haberstr. 7

69126 Heidelberg

Tel.: +49 6221-345-4303

Fax: +49 6221-345-4229

E-Mail: leserservice@springer.com

P.S. Vieles mehr rund um Ihr Fachgebiet finden Sie auf www.springermedizin.de 